\title{
ON APPROXIMATION SOLUTIONS OF THE CAUCHY-JENSEN AND THE ADDITIVE-QUADRATIC FUNCTIONAL EQUATION IN PARANORMED SPACES
}

\section{PRONDANAI KASKASEM ${ }^{1}$ AND CHAKKRID KLIN-EAM ${ }^{1,2, *}$}

${ }^{1}$ Department of Mathematics, Faculty of Science, Naresuan University, Phitsanulok, 65000, Thailand

${ }^{2}$ Research center for Academic Excellence in Mathematics, Naresuan University, Thailand

${ }^{*}$ Corresponding author: chakkridk@nu.ac.th

\section{Introduction and Preliminaries}

The stability problem of functional equations was initiated by Ulam in 1940 [17] arising from concerning the stability of group homomorphisms. These question form is the object of the stability theory. In 1941, Hyers [7] provided a first affirmative partial answer to Ulam's problem for the case of approximately additive mapping in Banach spaces. In 1978, Rassias [16] gave a generalization of Hyers's theorem for linear mapping by considering an unbounded Cauchy difference. A generalization of Rassias's result was developed by Găvruţa [6] in 1994 by replacing the unbounded Cauchy difference by a general control function. For more information on that subject and further references we refer to a survey paper [3] and to a recent monograph on Ulam stability [4].

Received 2018-12-21; accepted 2019-01-30; published 2019-05-01.

2010 Mathematics Subject Classification. 39B82; 39B52.

Key words and phrases. Hyers-Ulam-Rassias stability; bi-Cauchy-Jensen functional equation; bi-additive-quadratic functional equation; generalized Cauchy-Jensen functional equation; paranormed space.

(C) 2019 Authors retain the copyrights of their papers, and all open access articles are distributed under the terms of the Creative Commons Attribution License. 
Let $\mathbb{R}$ and $\mathbb{N}$ be the set of real numbers and the set of natural numbers, respectively. Next, let $X$ and $Y$ be vector spaces and $k$ be a positive integer, a function $f: X^{k} \rightarrow Y$ is called $k$-additive functional equation if and only if $f$ satisfies the equation

$$
\begin{aligned}
& f\left(x_{1}, x_{2}, \ldots, x_{i-1}, x_{i}+y, x_{i+1}, \ldots, x_{k}\right) \\
= & f\left(x_{1}, x_{1}, \ldots, x_{i}\right)+f\left(x_{1}, x_{2}, \ldots, x_{i-1}, y, x_{i+1}, \ldots, x_{k}\right)
\end{aligned}
$$

for all $i \in \mathbb{N}, 1 \leq i \leq k$ and for every $x_{1}, x_{2}, \ldots, x_{k}, y \in X$, that is, $f$ is additive in each of its variables $x_{i} \in X$ for all $i=1,2, \ldots, k$. Some fundamental properties on such mappings be mentioned in [10]. In particular, a 2-additive functional equation is called bi-additive functional equation.

A mapping $f: X \times X \rightarrow Y$ is called a bi-additive-quadratic functional equation (bi-AQE, shortly) if $f$ satisfies the system equations

$$
\begin{aligned}
f(x+y, z) & =f(x, z)+f(y, z), \\
f(x, y+z)+f(x, y-z) & =2 f(x, y)+2 f(x, z)
\end{aligned}
$$

for all $x, y, z \in X$. When $X=Y=\mathbb{R}$, the solution of (1.1) is given by the function $f(x, y)=c x y^{2}$ where $x, y, c \in \mathbb{R}$. For mapping $f: X \times X \rightarrow Y$ satisfies

$$
f(x+y, z+w)+f(x+y, z-w)=2 f(x, z)+2 f(x, w)+2 f(y, z)+2 f(y, w)
$$

for all $x, y, z, w \in X$. In 2005, Park, Bae and Chung [13] proved that the mapping $f: X \times X \rightarrow Y$ satisfies (1.1) if and only if it satisfies (1.2) and provided the general solution of (1.1) which is given by $f(x, y)=M(x, y, y)$ and $M(x, y, z)=M(x, z, y)$ for all $x, y, z \in X$ where $M: X \times X \times X \rightarrow Y$ is a multi-additive mapping.

A mapping $f: X \times X \rightarrow Y$ is called a bi-Cauchy-Jensen functional equation (bi-CJE, shortly) if $f$ satisfies the system equations

$$
\begin{aligned}
f(x+y, z) & =f(x, z)+f(y, z) \\
2 f\left(x, \frac{y+z}{2}\right) & =f(x, y)+f(x, z)
\end{aligned}
$$

for all $x, y, z \in X$. In particular, For $X=Y=\mathbb{R}$, The solution of (1.3) is given by the function $f(x, y)=$ $a x y+b x$ where $x, y, a, b \in \mathbb{R}$. For mapping $f: X \times X \rightarrow Y$ satisfies

$$
2 f\left(x+y, \frac{z+w}{2}\right)=f(x, z)+f(x, w)+f(y, z)+f(y, w)
$$

for all $x, y, z, w \in X$. In 2006, Park and Bae [12] showed that the mapping $f: X \times X \rightarrow Y$ satisfies (1.3) if and only if it satisfies (1.4) and gave the general solution of (1.4) which is given by $f(x, y)=B(x, y)+A(x)$ for all $x, y \in X$ where $B: X \times X \rightarrow Y$ is a bi-additive mapping and $A: X \rightarrow Y$ is an additive mapping. 
Next, we recall the concepts of paranormed space and some basic facts on the Fréchet spaces.

Definition 1.1 ([18]). Let $X$ be a vector space. A paranorm on $X$ is a function $P: X \rightarrow \mathbb{R}$ such that for all $x, y \in X$, the following conditions hold :

(i) $P(0)=0$;

(ii) $P(-x)=P(x)$;

(iii) $P(x+y) \leq P(x)+P(y)$ (triangle inequality);

(iv) If $\left\{t_{n}\right\}$ is a sequence of scalars with $t_{n} \rightarrow t$ and $\left\{x_{n}\right\} \subseteq X$ with $P\left(x_{n}-x\right) \rightarrow 0$, then $P\left(t_{n} x_{n}-t x\right) \rightarrow 0$. (continuity of scalar multication)

The pair $(X, P)$ is called a paranormed space if $P$ is a paranorm on $X$. Note that

$$
P(n x) \leq n P(x)
$$

for all $n \in \mathbb{N}$ and all $x \in X$. The paranorm $P$ on $X$ is called total if, in addition, $P$ satisfies

$$
\text { (v) } P(x)=0 \text { implies } x=0 .
$$

A Fréchet space is a total and complete paranormed space.

In 2015, Bae and Park [2] proved the Hyers-Ulam stability of the functional equation (1.2) and (1.4) in paranormed spaces in the sense of Rassias [16]. We refer to some works of stability of the functional equation (1.2) and (1.4) and various functional equations in paranormed spaces with [1,8,9,11,13-15]. In the first section of main results, we investigate stability of the functional equation (1.2) and (1.4) in paranormed spaces in the sense of Găvruţa [6].

In 2009, Gao et al. [5] introduced the generalized Cauchy-Jensen functional equation and gave some useful properties. Let $G$ be an $n$-divisible abelian group where $n \in \mathbb{N}$ and $X$ be a normed space with norm $\|\cdot\|_{X}$. For a mapping $f: G \rightarrow X$ is called a generalized Cauchy-Jensen functional equation (GCJE, shortly) if it satisfies the equation

$$
\alpha f\left(\frac{x+y}{\alpha}+z\right)=f(x)+f(y)+\alpha f(z)
$$

for all $x, y, z \in X$ and for any fixed positive integer $\alpha \geq 2$. In particular, when $\alpha=2$, it is called a Cauchy-Jensen functional equation (CJE, shortly).

Proposition $1.1([5])$. Let $G$ be an n-divisible abelian group for some positive integer $n$ and $X$ be a normed space with norm $\|\cdot\|_{X}$. Then a mapping $f: G \rightarrow X$ is additive if and only if it satisfies

$$
\|f(x)+f(y)+n f(z)\|_{X} \leq\left\|n f\left(\frac{x+y}{n}+z\right)\right\|_{X}
$$

for all $x, y, z \in G$.

The following corollary is an immediate consequence of Proposition 1.1. 
Corollary 1.1 ([5]). For a mapping $f: G \rightarrow X$, the following statements are equivalent.
(a) $f$ is additive.
(b) $f(x)+f(y)+n f(z)=n f\left(\frac{x+y}{n}+z\right)$, for all $x, y, z \in G$.
(c) $\|f(x)+f(y)+n f(z)\|_{X} \leq\left\|n f\left(\frac{x+y}{n}+z\right)\right\|_{X}$, for all $x, y, z \in G$.

Clearly, a vector space is $n$-divisible abelian group, so Corollary 1.1 is right when $G$ is a vector space. In the second section of main results, we proved the stability of the functional equation (1.5) in paranormed spaces in the sense of Rassias [16].

Throughtout this paper, assume that $(X, P)$ is a Fréchet space and that $(E,\|\cdot\|)$ is a Banach space.

2. The Stability of the Bi-Cauchy-Jensen functional EQUation And BI-AdDitive-Quadratic FUNCTIONAL EQUATION IN PARANORMED SPACES

The following result is the generalized Hyers-Ulam-Rassias stability of the functional equation (1.4).

Theorem 2.1. Let $\varphi: E \times E \times E \times E \rightarrow[0, \infty)$ be a function and $f: E \times E \rightarrow X$ be a mapping satisfying $f(x, 0)=0$ for all $x \in E$ such that

$$
\begin{aligned}
& P\left(2 f\left(x+y, \frac{z+w}{2}\right)-f(x, z)-f(x, w)-f(y, z)-f(y, w)\right) \\
\leq & \varphi(x, y, z, w)
\end{aligned}
$$

for all $x, y, z, w \in E$. Then there exists a unique mapping $F: E \times E \rightarrow X$ satisfying (1.4) such that

$$
P(2 f(x, y)-F(x, y)) \leq \tilde{\varphi}(x, x, y, y)
$$

for all $x, y \in E$ where

$$
\begin{aligned}
& \tilde{\varphi}(x, y, z, w) \\
:= & \sum_{j=0}^{\infty} 6^{j}\left[6 \varphi\left(\frac{x}{2^{j+1}}, \frac{y}{2^{j+1}}, \frac{z}{3^{j+1}},-\frac{w}{3^{j+1}}\right)+4 \varphi\left(\frac{x}{2^{j+1}}, \frac{y}{2^{j+1}},-\frac{z}{3^{j+1}}, \frac{w}{3^{j+1}}\right)\right. \\
& +2 \varphi\left(\frac{x}{2^{j+1}}, \frac{y}{2^{j+1}}, \frac{z}{3^{j+1}}, \frac{w}{3^{j+1}}\right)+2 \varphi\left(\frac{x}{2^{j+1}}, \frac{y}{2^{j+1}},-\frac{z}{3^{j+1}}, \frac{w}{3^{j}}\right) \\
& \left.+\varphi\left(\frac{x}{2^{j+1}}, \frac{y}{2^{j+1}}, \frac{z}{3^{j}}, \frac{w}{3^{j}}\right)\right]<\infty
\end{aligned}
$$

for all $x, y, z, w \in E$ and the mapping $F: E \times E \rightarrow X$ is given by

$$
F(x, y)=\lim _{j \rightarrow \infty} 2 \cdot 6^{j} f\left(\frac{x}{2^{j}}, \frac{y}{3^{j}}\right)
$$

for all $x, y \in E$. 
Proof. Letting $y=x$ in (2.1), we obtain that

$$
P\left(2 f\left(2 x, \frac{z+w}{2}\right)-2 f(x, z)-2 f(x, w)\right) \leq \varphi(x, x, z, w)
$$

for all $x, z, w \in E$. Letting $w=-z$ in (2.4), we get that

$$
P(2 f(x, z)+2 f(x,-z)) \leq \varphi(x, x, z,-z)
$$

for all $x, z \in E$. Subtituting $z$ by $-z$ and $w$ by $-z$ in (2.4), we get

$$
P(2 f(2 x,-z)-4 f(x,-z)) \leq \varphi(x, x,-z,-z)
$$

for all $x, z \in E$. It follows from (2.5) and (2.6) that

$$
\begin{aligned}
& P(4 f(x, z)+2 f(2 x,-z)) \\
& =P(4 f(x, z)+4 f(x,-z)-4 f(x,-z)+2 f(2 x,-z)) \\
& \leq P(4 f(x, z)+4 f(x,-z))+P(2 f(2 x,-z)-4 f(x,-z)) \\
& \leq 2 P(2 f(x, z)+2 f(x,-z))+P(2 f(2 x,-z)-4 f(x,-z)) \\
& \leq 2 \varphi(x, x, z,-z)+\varphi(x, x,-z,-z)
\end{aligned}
$$

for all $x, z \in E$. Letting $w=-3 z$ in (2.4), we have

$$
P(2 f(2 x,-z)-2 f(x,-3 z)-2 f(x, z)) \leq \varphi(x, x, z,-3 z)
$$

for all $x, z \in E$. By $(i i)$ of definition 1.1, we have

$$
P(2 f(x,-3 z)+2 f(x, z)-2 f(2 x,-z)) \leq \varphi(x, x, z,-3 z)
$$

for all $x, z \in E$. By (2.7) and (2.8), we have

$$
\begin{aligned}
& P(6 f(x, z)+2 f(x,-3 z)) \\
= & P(4 f(x, z)+2 f(2 x,-z)+2 f(x,-3 z)+2 f(x, z)-2 f(2 x,-z)) \\
\leq & P(4 f(x, z)+2 f(2 x,-z))+P(2 f(x,-3 z)+2 f(x, z)-2 f(2 x,-z)) \\
\leq & 2 \varphi(x, x, z,-z)+\varphi(x, x,-z,-z)+\varphi(x, x, z,-3 z)
\end{aligned}
$$

for all $x, z \in E$. Putting $z=3 z$ in (2.6), we obtain that

$$
P(2 f(2 x,-3 z)-4 f(x,-3 z)) \leq \varphi(x, x,-3 z,-3 z)
$$


for all $x, z \in E$. It follows from (2.9) and (2.10)

$$
\begin{aligned}
& P(12 f(x, z)+2 f(2 x,-3 z)) \\
= & P(12 f(x, z)+4 f(x,-3 z)-4 f(x,-3 z)+2 f(2 x,-3 z)) \\
\leq & P(12 f(x, z)+4 f(x,-3 z))+P(2 f(2 x,-3 z)-4 f(x,-3 z)) \\
\leq & 2 P(6 f(x, z)+2 f(x,-3 z))+P(2 f(2 x,-3 z)-4 f(x,-3 z)) \\
\leq & 4 \varphi(x, x, z,-z)+2 \varphi(x, x,-z,-z)+2 \varphi(x, x, z,-3 z) \\
& +\varphi(x, x,-3 z,-3 z)
\end{aligned}
$$

for all $x, z \in E$. Replacing $z$ by $-z$ in the above inequality, we get that

$$
\begin{aligned}
& P(12 f(x,-z)+2 f(2 x, 3 z)) \\
\leq & 4 \varphi(x, x,-z, z)+2 \varphi(x, x, z, z)+2 \varphi(x, x,-z, 3 z)+\varphi(x, x, 3 z, 3 z)
\end{aligned}
$$

for all $x, z \in E$. By (2.5) and the above inequality, we have

$$
\begin{aligned}
& P(12 f(x, z)-2 f(2 x, 3 z)) \\
= & P(12 f(x, z)+12 f(x,-z)-12 f(x,-z)-2 f(2 x, 3 z)) \\
\leq & P(12 f(x, z)+12 f(x,-z))+P(-12 f(x,-z)-2 f(2 x, 3 z)) \\
= & P(12 f(x, z)+12 f(x,-z))+P(12 f(x,-z)+2 f(2 x, 3 z)) \\
\leq & 6 P(2 f(x, z)+2 f(x,-z))+P(12 f(x,-z)+2 f(2 x, 3 z)) \\
\leq & 6 \varphi(x, x, z,-z)+4 \varphi(x, x,-z, z)+2 \varphi(x, x, z, z) \\
& +2 \varphi(x, x,-z, 3 z)+\varphi(x, x, 3 z, 3 z)
\end{aligned}
$$

for all $x, z \in E$. Replacing $x$ by $\frac{x}{2^{j+1}}$ and $z$ by $\frac{z}{3^{j+1}}$ in (2.13), we obtain that

$$
\begin{aligned}
& P\left(12 f\left(\frac{x}{2^{j+1}}, \frac{z}{3^{j+1}}\right)-2 f\left(\frac{x}{2^{j}}, \frac{z}{3^{j}}\right)\right) \\
\leq & 6 \varphi\left(\frac{x}{2^{j+1}}, \frac{x}{2^{j+1}}, \frac{z}{3^{j+1}},-\frac{z}{3^{j+1}}\right)+4 \varphi\left(\frac{x}{2^{j+1}}, \frac{x}{2^{j+1}},-\frac{z}{3^{j+1}}, \frac{z}{3^{j+1}}\right) \\
& +2 \varphi\left(\frac{x}{2^{j+1}}, \frac{x}{2^{j+1}}, \frac{z}{3^{j+1}}, \frac{z}{3^{j+1}}\right)+2 \varphi\left(\frac{x}{2^{j+1}}, \frac{x}{2^{j+1}},-\frac{z}{3^{j+1}}, \frac{z}{3^{j}}\right) \\
& +\varphi\left(\frac{x}{2^{j+1}}, \frac{x}{2^{j+1}}, \frac{z}{3^{j}}, \frac{z}{3^{j}}\right)
\end{aligned}
$$


for all $x, z \in E$. By (2.14), for any integers $l, m$ such that $0 \leq l<m$, we get that

$$
\begin{aligned}
& P\left(2 \cdot 6^{m} f\left(\frac{x}{2^{m}}, \frac{z}{3^{m}}\right)-2 \cdot 6^{l} f\left(\frac{x}{2^{l}}, \frac{z}{3^{l}}\right)\right) \\
& =P\left(2 \cdot 6^{m} f\left(\frac{x}{2^{m}}, \frac{z}{3^{m}}\right)-2 \cdot 6^{m-1} f\left(\frac{x}{2^{m-1}}, \frac{z}{3^{m-1}}\right)+2 \cdot 6^{m-1} f\left(\frac{x}{2^{m-1}}, \frac{z}{3^{m-1}}\right)\right. \\
& -2 \cdot 6^{m-2} f\left(\frac{x}{2^{m-2}}, \frac{z}{3^{m-2}}\right)+2 \cdot 6^{m-2} f\left(\frac{x}{2^{m-2}}, \frac{z}{3^{m-2}}\right) \\
& \left.+\cdots+2 \cdot 6^{l+1} f\left(\frac{x}{2^{l+1}}, \frac{z}{3^{l+1}}\right)-2 \cdot 6^{l} f\left(\frac{x}{2^{l}}, \frac{z}{3^{l}}\right)\right) \\
& \leq P\left(2 \cdot 6^{m} f\left(\frac{x}{2^{m}}, \frac{z}{3^{m}}\right)-2 \cdot 6^{m-1} f\left(\frac{x}{2^{m-1}}, \frac{z}{3^{m-1}}\right)\right) \\
& +P\left(2 \cdot 6^{m-1} f\left(\frac{x}{2^{m-1}}, \frac{z}{3^{m-1}}\right)-2 \cdot 6^{m-2} f\left(\frac{x}{2^{m-2}}, \frac{z}{3^{m-2}}\right)\right) \\
& +\cdots+P\left(2 \cdot 6^{l+1} f\left(\frac{x}{2^{l+1}}, \frac{z}{3^{l+1}}\right)-2 \cdot 6^{l} f\left(\frac{x}{2^{l}}, \frac{z}{3^{l}}\right)\right) \\
& \leq 6^{m-1} P\left(12 f\left(\frac{x}{2^{m}}, \frac{z}{3^{m}}\right)-2 f\left(\frac{x}{2^{m-1}}, \frac{z}{3^{m-1}}\right)\right) \\
& +6^{m-2} P\left(12 f\left(\frac{x}{2^{m-1}}, \frac{z}{3^{m-1}}\right)-2 f\left(\frac{x}{2^{m-2}}, \frac{z}{3^{m-2}}\right)\right) \\
& +\cdots+6^{l} P\left(12 f\left(\frac{x}{2^{l+1}}, \frac{z}{3^{l+1}}\right)-2 f\left(\frac{x}{2^{l}}, \frac{z}{3^{l}}\right)\right) \\
& =\sum_{j=l}^{m-1} 6^{j} P\left(12 f\left(\frac{x}{2^{j+1}}, \frac{z}{3^{j+1}}\right)-2 f\left(\frac{x}{2^{j}}, \frac{z}{3^{j}}\right)\right) \\
& \leq \sum_{j=l}^{\infty} 6^{j}\left[6 \varphi\left(\frac{x}{2^{j+1}}, \frac{x}{2^{j+1}}, \frac{z}{3^{j+1}},-\frac{z}{3^{j+1}}\right)+4 \varphi\left(\frac{x}{2^{j+1}}, \frac{x}{2^{j+1}},-\frac{z}{3^{j+1}}, \frac{z}{3^{j+1}}\right)\right. \\
& +2 \varphi\left(\frac{x}{2^{j+1}}, \frac{x}{2^{j+1}}, \frac{z}{3^{j+1}}, \frac{z}{3^{j+1}}\right)+2 \varphi\left(\frac{x}{2^{j+1}}, \frac{x}{2^{j+1}},-\frac{z}{3^{j+1}}, \frac{z}{3^{j}}\right) \\
& \left.+\varphi\left(\frac{x}{2^{j+1}}, \frac{x}{2^{j+1}}, \frac{z}{3^{j}}, \frac{z}{3^{j}}\right)\right]
\end{aligned}
$$

for all $x, z \in E$. It follows from (2.3) that

$$
\begin{aligned}
& \lim _{l \rightarrow \infty} \sum_{j=l}^{\infty} 6^{j}\left[6 \varphi\left(\frac{x}{2^{j+1}}, \frac{x}{2^{j+1}}, \frac{z}{3^{j+1}},-\frac{z}{3^{j+1}}\right)+4 \varphi\left(\frac{x}{2^{j+1}}, \frac{x}{2^{j+1}},-\frac{z}{3^{j+1}}, \frac{z}{3^{j+1}}\right)\right. \\
& +2 \varphi\left(\frac{x}{2^{j+1}}, \frac{x}{2^{j+1}}, \frac{z}{3^{j+1}}, \frac{z}{3^{j+1}}\right)+2 \varphi\left(\frac{x}{2^{j+1}}, \frac{x}{2^{j+1}},-\frac{z}{3^{j+1}}, \frac{z}{3^{j}}\right) \\
& \left.+\varphi\left(\frac{x}{2^{j+1}}, \frac{x}{2^{j+1}}, \frac{z}{3^{j}}, \frac{z}{3^{j}}\right)\right]=0
\end{aligned}
$$

for all $x, z \in E$. This implies that the sequence $\left\{2 \cdot 6^{j} f\left(\frac{x}{2^{j}}, \frac{z}{3^{j}}\right)\right\}_{j=0}^{\infty}$ is a Cauchy sequence in $X$ for all $x, z \in E$. Since $X$ is complete paranormed space, the sequence $\left\{2 \cdot 6^{j} f\left(\frac{x}{2^{j}}, \frac{z}{3^{j}}\right)\right\}_{j=0}^{\infty}$ converges for all $x, z \in E$. Define $F: E \times E \rightarrow X$ by

$$
F(x, z)=\lim _{j \rightarrow \infty} 2 \cdot 6^{j} f\left(\frac{x}{2^{j}}, \frac{z}{3^{j}}\right)
$$


for all $x, z \in E$. By (2.3), we get that

$$
\begin{aligned}
& \sum_{j=1}^{\infty} \frac{1}{6} \cdot 2 \cdot 6^{j} \varphi\left(\frac{x}{2^{j}}, \frac{y}{2^{j}}, \frac{z}{3^{j}}, \frac{w}{3^{j}}\right) \\
= & \sum_{j=0}^{\infty} \frac{1}{6} \cdot 2^{\cdot} 6^{j+1} \varphi\left(\frac{x}{2^{j+1}}, \frac{y}{2^{j+1}}, \frac{z}{3^{j+1}}, \frac{w}{3^{j+1}}\right) \\
= & \sum_{j=0}^{\infty} 2 \cdot 6^{j} \varphi\left(\frac{x}{2^{j+1}}, \frac{y}{2^{j+1}}, \frac{z}{3^{j+1}}, \frac{w}{3^{j+1}}\right) \\
\leq & \sum_{j=0}^{\infty} 6 \cdot 6^{j} \varphi\left(\frac{x}{2^{j+1}}, \frac{y}{2^{j+1}}, \frac{z}{3^{j+1}},-\frac{w}{3^{j+1}}\right)+4 \cdot \sum_{j=0}^{\infty} 6^{j} \varphi\left(\frac{x}{2^{j+1}}, \frac{y}{2^{j+1}},-\frac{z}{3^{j+1}}, \frac{w}{3^{j+1}}\right) \\
& +2 \cdot \sum_{j=0}^{\infty} 6^{j} \varphi\left(\frac{x}{2^{j+1}}, \frac{y}{2^{j+1}}, \frac{z}{3^{j+1}}, \frac{w}{3^{j+1}}\right)+2 \cdot \sum_{j=0}^{\infty} 6^{j} \varphi\left(\frac{x}{2^{j+1}}, \frac{y}{2^{j+1}},-\frac{z}{3^{j+1}}, \frac{w}{3^{j}}\right) \\
& +\sum_{j=0}^{\infty} 6^{j} \varphi\left(\frac{x}{2^{j+1}}, \frac{y}{2^{j+1}}, \frac{z}{3^{j}}, \frac{w}{3^{j}}\right) \\
\leq & \sum_{j=0}^{\infty} 6^{j}\left[6 \varphi\left(\frac{x}{2^{j+1}}, \frac{y}{2^{j+1}}, \frac{z}{3^{j+1}},-\frac{w}{3^{j+1}}\right)+4 \varphi\left(\frac{x}{2^{j+1}}, \frac{y}{2^{j+1}},-\frac{z}{3^{j+1}}, \frac{w}{3^{j+1}}\right)\right. \\
& +2 \varphi\left(\frac{x}{2^{j+1}}, \frac{y}{2^{j+1}}, \frac{z}{3^{j+1}}, \frac{w}{3^{j+1}}\right)+2 \varphi\left(\frac{x}{2^{j+1}}, \frac{y}{2^{j+1}},-\frac{z}{3^{j+1}}, \frac{w}{3^{j}}\right) \\
& \left.+\varphi\left(\frac{x}{2^{j+1}}, \frac{y}{2^{j+1}}, \frac{z}{3^{j}}, \frac{w}{3^{j}}\right)\right] \\
= & \tilde{\varphi}(x, y, z, w)<\infty
\end{aligned}
$$

for all $x, y, z, w \in E$. This implies that

$$
\lim _{j \rightarrow \infty} \frac{1}{6} \cdot 2 \cdot 6^{j} \varphi\left(\frac{x}{2^{j}}, \frac{y}{2^{j}}, \frac{z}{3^{j}}, \frac{w}{3^{j}}\right)=0
$$

for all $x, y, z, w \in E$, which implies

$$
\lim _{j \rightarrow \infty} 2 \cdot 6^{j} \varphi\left(\frac{x}{2^{j}}, \frac{y}{2^{j}}, \frac{z}{3^{j}}, \frac{w}{3^{j}}\right)=0
$$

for all $x, y, z, w \in E$. It follows from (2.1), (2.16) and (2.17) that we have

$$
\begin{aligned}
& P\left(2 F\left(x+y, \frac{z+w}{2}\right)-F(x, z)-F(x, w)-F(y, z)-F(y, w)\right) \\
\leq & P\left(2 \lim _{j \rightarrow \infty} 2 \cdot 6^{j} f\left(\frac{x+y}{2^{j}}, \frac{\frac{z+w}{2}}{3^{j}}\right)-\lim _{j \rightarrow \infty} 2 \cdot 6^{j} f\left(\frac{x}{2^{j}}, \frac{z}{3^{j}}\right)\right. \\
& \left.-\lim _{j \rightarrow \infty} 2 \cdot 6^{j} f\left(\frac{x}{2^{j}}, \frac{w}{3^{j}}\right)-\lim _{j \rightarrow \infty} 2 \cdot 6^{j} f\left(\frac{y}{2^{j}}, \frac{z}{3^{j}}\right)-\lim _{j \rightarrow \infty} 2 \cdot 6^{j} f\left(\frac{y}{2^{j}}, \frac{w}{3^{j}}\right)\right) \\
\leq & \lim _{j \rightarrow \infty} 2 \cdot 6^{j} P\left(2 f\left(\frac{x}{2^{j}}+\frac{y}{2^{j}}, \frac{\frac{z}{3^{j}}+\frac{w}{3^{j}}}{2}\right)-f\left(\frac{x}{2^{j}}, \frac{z}{3^{j}}\right)-f\left(\frac{x}{2^{j}}, \frac{w}{3^{j}}\right)\right. \\
& \left.-f\left(\frac{y}{2^{j}}, \frac{z}{3^{j}}\right)-f\left(\frac{y}{2^{j}}, \frac{w}{3^{j}}\right)\right) \\
\leq & \lim _{j \rightarrow \infty} 2 \cdot 6^{j} \varphi\left(\frac{x}{2^{j}}, \frac{y}{2^{j}}, \frac{z}{3^{j}}, \frac{w}{3^{j}}\right)=0
\end{aligned}
$$


for all $x, y, z, w \in E$. Since $X$ is total, we have

$$
2 F\left(x+y, \frac{z+w}{2}\right)=F(x, z)+F(x, w)+F(y, z)+F(y, w)
$$

for all $x, y, z, w \in E$. Setting $l=0$ and taking $m \rightarrow \infty$ in (2.15), this implies that the inequality (2.2). Next, we will show that $F$ is unique. Let $G: E \times E \rightarrow X$ be another mapping satisfying (1.4) and (2.2). By [12], there exists bi-additive mapping $B, B^{\prime}: E \times E \rightarrow X$ and additive mapping $A, A^{\prime}: E \rightarrow X$ such that $F(x, y)=B(x, y)+A(x)$ and $G(x, y)=B^{\prime}(x, y)+A^{\prime}(x)$ for all $x, y \in E$. Since $B$ is bi-additive mapping, $A$ is additive mapping and $f(x, 0)=0$ for all $x \in E$, we have

$$
\begin{aligned}
F(x, y)-6 F\left(\frac{x}{2}, \frac{x}{3}\right) & =[B(x, y)+A(x)]-6\left[B\left(\frac{x}{2}, \frac{y}{3}\right)+A\left(\frac{x}{2}\right)\right] \\
& =B(x, y)+A(x)-6 B\left(\frac{x}{2}, \frac{y}{3}\right)-6 A\left(\frac{x}{2}\right) \\
& =B(x, y)+A(x)-B(x, y)-3 A(x) \\
& =-2 A(x) \\
& =-2 B(x, 0)-2 A(x) \\
& =-2 F(x, 0) \\
& =-2 \lim _{j \rightarrow \infty} 2 \cdot 6^{j} f\left(\frac{x}{2^{j}}, 0\right)=0
\end{aligned}
$$

for all $x, y \in E$, that is,

$$
F(x, y)=6 F\left(\frac{x}{2}, \frac{y}{3}\right)
$$

for all $x, y \in E$. Replacing $x$ by $\frac{x}{2}$ and $y$ by $\frac{y}{3}$ in (2.18), we have

$$
F\left(\frac{x}{2}, \frac{y}{3}\right)=6 F\left(\frac{x}{2^{2}}, \frac{y}{3^{2}}\right)
$$

for all $x, y \in E$. Continuing this process, we have $F(x, y)=6^{n} F\left(\frac{x}{2^{n}}, \frac{y}{3^{n}}\right)$ for all $x, y \in E$ and for all $n \in \mathbb{N}$. Similarly, we get that $G(x, y)=6^{n} G\left(\frac{x}{2^{n}}, \frac{y}{3^{n}}\right)$ for all $x, y \in E$ and for all $n \in \mathbb{N}$. For any $n \in \mathbb{N}$, we obtain 
that

$$
\begin{aligned}
& P(F(x, y)-G(x, y)) \\
& =P\left(6^{n} F\left(\frac{x}{2^{n}}, \frac{y}{3^{n}}\right)-6^{n} G\left(\frac{x}{2^{n}}, \frac{y}{3^{n}}\right)\right) \\
& =P\left(6^{n} F\left(\frac{x}{2^{n}}, \frac{y}{3^{n}}\right)-2 \cdot 6^{n} f\left(\frac{x}{2^{n}}, \frac{y}{3^{n}}\right)+2 \cdot 6^{n} f\left(\frac{x}{2^{n}}, \frac{y}{3^{n}}\right)-6^{n} G\left(\frac{x}{2^{n}}, \frac{y}{3^{n}}\right)\right) \\
& \leq P\left(6^{n} F\left(\frac{x}{2^{n}}, \frac{y}{3^{n}}\right)-2 \cdot 6^{n} f\left(\frac{x}{2^{n}}, \frac{y}{3^{n}}\right)\right)+P\left(2 \cdot 6^{n} f\left(\frac{x}{2^{n}}, \frac{y}{3^{n}}\right)-6^{n} G\left(\frac{x}{2^{n}}, \frac{y}{3^{n}}\right)\right) \\
& \leq 6^{n} P\left(F\left(\frac{x}{2^{n}}, \frac{y}{3^{n}}\right)-2 f\left(\frac{x}{2^{n}}, \frac{y}{3^{n}}\right)\right)+6^{n} P\left(2 f\left(\frac{x}{2^{n}}, \frac{y}{3^{n}}\right)-G\left(\frac{x}{2^{n}}, \frac{y}{3^{n}}\right)\right) \\
& \leq 2 \cdot 6^{n} \tilde{\varphi}\left(\frac{x}{2^{n}}, \frac{x}{2^{n}}, \frac{y}{3^{n}}, \frac{y}{3^{n}}\right) \\
& =2 \cdot 6^{n} \sum_{j=0}^{\infty} 6^{j}\left[6 \varphi\left(\frac{\frac{x}{2^{n}}}{2^{j+1}}, \frac{\frac{x}{2^{n}}}{2^{j+1}}, \frac{\frac{y}{3^{n}}}{3^{j+1}},-\frac{\frac{y}{3^{n}}}{3^{j+1}}\right)+4 \varphi\left(\frac{\frac{x}{2^{n}}}{2^{j+1}}, \frac{\frac{x}{2^{n}}}{2^{j+1}},-\frac{\frac{y}{3^{n}}}{3^{j+1}}, \frac{\frac{y}{3^{n}}}{3^{j+1}}\right)\right. \\
& +2 \varphi\left(\frac{\frac{x}{2^{n}}}{2^{j+1}}, \frac{\frac{x}{2^{n}}}{2^{j+1}}, \frac{\frac{y}{3^{n}}}{3^{j+1}}, \frac{\frac{y}{3^{n}}}{3^{j+1}}\right)+2 \varphi\left(\frac{\frac{x}{2^{n}}}{2^{j+1}}, \frac{\frac{x}{2^{n}}}{2^{j+1}},-\frac{\frac{y}{3^{n}}}{3^{j+1}}, \frac{\frac{y}{3^{n}}}{3^{j}}\right) \\
& \left.+\varphi\left(\frac{\frac{x}{2^{n}}}{2^{j+1}}, \frac{\frac{x}{2^{n}}}{2^{j+1}}, \frac{\frac{y}{3^{n}}}{3^{j}}, \frac{\frac{y}{3^{n}}}{3^{j}}\right)\right] \\
& =2 \sum_{j=0}^{\infty} 6^{n+j}\left[6 \varphi\left(\frac{x}{2^{n+j+1}}, \frac{x}{2^{n+j+1}}, \frac{y}{3^{n+j+1}},-\frac{y}{3^{n+j+1}}\right)\right. \\
& +4 \varphi\left(\frac{x}{2^{n+j+1}}, \frac{x}{2^{n+j+1}},-\frac{y}{3^{n+j+1}}, \frac{y}{3^{n+j+1}}\right)+2 \varphi\left(\frac{x}{2^{n+j+1}}, \frac{x}{2^{n+j+1}}, \frac{y}{3^{n+j+1}}, \frac{y}{3^{n+j+1}}\right) \\
& \left.+2 \varphi\left(\frac{x}{2^{n+j+1}}, \frac{x}{2^{n+j+1}},-\frac{y}{3^{n+j+1}}, \frac{y}{3^{n+j}}\right)+\varphi\left(\frac{x}{2^{n+j+1}}, \frac{x}{2^{n+j+1}}, \frac{y}{3^{n+j}}, \frac{y}{3^{n+j}}\right)\right] \\
& =2 \sum_{i=n}^{\infty} 6^{i}\left[6 \varphi\left(\frac{x}{2^{i+1}}, \frac{x}{2^{i+1}}, \frac{y}{3^{i+1}},-\frac{y}{3^{i+1}}\right)+4 \varphi\left(\frac{x}{2^{i+1}}, \frac{x}{2^{i+1}},-\frac{y}{3^{i+1}}, \frac{y}{3^{i+1}}\right)\right. \\
& +2 \varphi\left(\frac{x}{2^{i+1}}, \frac{x}{2^{i+1}}, \frac{y}{3^{i+1}}, \frac{y}{3^{i+1}}\right)+2 \varphi\left(\frac{x}{2^{i+1}}, \frac{x}{2^{i+1}},-\frac{y}{3^{i+1}}, \frac{y}{3^{i}}\right) \\
& \left.+\varphi\left(\frac{x}{2^{i+1}}, \frac{x}{2^{i+1}}, \frac{y}{3^{i}}, \frac{y}{3^{i}}\right)\right]
\end{aligned}
$$

for all $x, y \in E$. By (2.3), we obtain that

$$
\begin{aligned}
& \lim _{n \rightarrow \infty} \sum_{i=n}^{\infty} 6^{i}\left[6 \varphi\left(\frac{x}{2^{i+1}}, \frac{x}{2^{i+1}}, \frac{y}{3^{i+1}},-\frac{y}{3^{i+1}}\right)+4 \varphi\left(\frac{x}{2^{i+1}}, \frac{x}{2^{i+1}},-\frac{y}{3^{i+1}}, \frac{y}{3^{i+1}}\right)\right. \\
& +2 \varphi\left(\frac{x}{2^{i+1}}, \frac{x}{2^{i+1}}, \frac{y}{3^{i+1}}, \frac{y}{3^{i+1}}\right)+2 \varphi\left(\frac{x}{2^{i+1}}, \frac{x}{2^{i+1}},-\frac{y}{3^{i+1}}, \frac{y}{3^{i}}\right) \\
& \left.+\varphi\left(\frac{x}{2^{i+1}}, \frac{x}{2^{i+1}}, \frac{y}{3^{i}}, \frac{y}{3^{i}}\right)\right]=0
\end{aligned}
$$

for all $x, y \in E$. From (2.20), taking limit $n \rightarrow \infty$ in (2.19), we obtain that

$$
\lim _{n \rightarrow \infty} P(F(x, y)-G(x, y))=0
$$

for all $x, y \in E$. Since paranorm $P$ on $X$ is total, we have $F(x, y)-G(x, y)=0$ for all $x, y \in E$. Hence $F$ is a unique mapping satisfying (1.4) and (2.2). 
Remark 2.1. Let $r, \theta$ be positive real numbers with $r>\log _{2} 6$. If we set $\varphi(x, y, z, w)=\theta\left(\|x\|^{r}+\|y\|^{r}+\right.$ $\left.\|z\|^{r}+\|w\|^{r}\right)$ for all $x, y, z, w \in E$, then Theorem 2.1 recovers Theorem 2.1 in [2].

The following result is the generalized Hyers-Ulam-Rassias stability of the functional equation (1.2).

Theorem 2.2. Let $\varphi: E \times E \times E \times E \rightarrow[0, \infty)$ be a function and $f: E \times E \rightarrow X$ be a mapping satisfying $f(x, 0)=0$ for all $x \in E$ such that

$$
\begin{aligned}
& P(f(x+y, z+w)+f(x+y, z-w)-2 f(x, z)-2 f(x, w)-2 f(y, z)-2 f(y, w)) \\
\leq & \varphi(x, y, z, w)
\end{aligned}
$$

for all $x, y, z, w \in E$. Then there exists a unique mapping $F: E \times E \rightarrow X$ satisfying (1.2) such that

$$
P(f(x, y)-F(x, y)) \leq \tilde{\varphi}(x, x, y, y)
$$

for all $x, y \in E$ where

$$
\tilde{\varphi}(x, y, z, w):=\sum_{j=0}^{\infty} 8^{j} \varphi\left(\frac{x}{2^{j+1}}, \frac{y}{2^{j+1}}, \frac{z}{2^{j+1}}, \frac{w}{2^{j+1}}\right)<\infty
$$

for all $x, y, z, w \in E$ where the mapping $F: E \times E \rightarrow X$ is given by

$$
F(x, y)=\lim _{j \rightarrow \infty} 8^{j} f\left(\frac{x}{2^{j}}, \frac{y}{2^{j}}\right)
$$

for all $x, y \in E$.

Proof. Letting $y=x$ and $w=z$ in (2.21), we obtain that

$$
P(f(2 x, 2 z)-8 f(x, z)) \leq \varphi(x, x, z, z)
$$

for all $x, z \in E$. Replacing $x$ by $\frac{x}{2^{j+1}}$ and $z$ by $\frac{z}{2^{j+1}}$ in the above inequality, we get that

$$
P\left(f\left(\frac{x}{2^{j}}, \frac{z}{2^{j}}\right)-8 f\left(\frac{x}{2^{j+1}}, \frac{z}{2^{j+1}}\right)\right) \leq \varphi\left(\frac{x}{2^{j+1}}, \frac{x}{2^{j+1}}, \frac{z}{2^{j+1}}, \frac{z}{2^{j+1}}\right)
$$

for all nonnegative integer $j$ and for all $x, z \in E$. It follows from (2.24) that we have

$$
\begin{aligned}
& P\left(8^{j} f\left(\frac{x}{2^{j}}, \frac{z}{2^{j}}\right)-8^{j+1} f\left(\frac{x}{2^{j+1}}, \frac{z}{2^{j+1}}\right)\right) \\
\leq & 8^{j} P\left(f\left(\frac{x}{2^{j}}, \frac{z}{2^{j}}\right)-8 f\left(\frac{x}{2^{j+1}}, \frac{z}{2^{j+1}}\right)\right) \\
\leq & 8^{j} \varphi\left(\frac{x}{2^{j+1}}, \frac{x}{2^{j+1}}, \frac{z}{2^{j+1}}, \frac{z}{2^{j+1}}\right)
\end{aligned}
$$


for all nonnegative integer $j$ and for all $x, z \in E$. By (2.25), for any integers $l$ and $m$ such that $0 \leq l<m$, we have

$$
\begin{aligned}
& P\left(8^{l} f\left(\frac{x}{2^{l}}, \frac{y}{2^{l}}\right)-8^{m} f\left(\frac{x}{2^{m}}, \frac{y}{2^{m}}\right)\right) \\
= & P\left(8^{l} f\left(\frac{x}{2^{l}}, \frac{y}{2^{l}}\right)-8^{l+1} f\left(\frac{x}{2^{l+1}}, \frac{y}{2^{l+1}}\right)+8^{l+1} f\left(\frac{x}{2^{l+1}}, \frac{y}{2^{l+1}}\right)\right. \\
& \left.+\cdots+8^{m-1} f\left(\frac{x}{2^{m-1}}, \frac{y}{2^{m-1}}\right)-8^{m} f\left(\frac{x}{2^{m}}, \frac{y}{2^{m}}\right)\right) \\
\leq & P\left(8^{l} f\left(\frac{x}{2^{l}}, \frac{y}{2^{l}}\right)-8^{l+1} f\left(\frac{x}{2^{l+1}}, \frac{y}{2^{l+1}}\right)\right) \\
& +P\left(8^{l+1} f\left(\frac{x}{2^{l+1}}, \frac{y}{2^{l+1}}\right)-8^{l+2} f\left(\frac{x}{2^{l+2}}, \frac{y}{2^{l+2}}\right)\right) \\
& +\cdots+P\left(8^{m-1} f\left(\frac{x}{2^{m-1}}, \frac{y}{2^{m-1}}\right)-8^{m} f\left(\frac{x}{2^{m}}, \frac{y}{2^{m}}\right)\right) \\
= & \sum_{j=l}^{m-1} 8^{j} \varphi\left(\frac{x}{2^{j+1}}, \frac{x}{2^{j+1}}, \frac{z}{2^{j+1}}, \frac{z}{2^{j+1}}\right) \\
\leq & \sum_{j=l}^{\infty} 8^{j} \varphi\left(\frac{x}{2^{j+1}}, \frac{x}{2^{j+1}}, \frac{z}{2^{j+1}}, \frac{z}{2^{j+1}}\right)
\end{aligned}
$$

for all $x, z \in E$. It follows from (2.23) that we obtain that

$$
\lim _{l \rightarrow \infty} \sum_{j=l}^{\infty} 8^{j} \varphi\left(\frac{x}{2^{j+1}}, \frac{x}{2^{j+1}}, \frac{z}{2^{j+1}}, \frac{z}{2^{j+1}}\right)=0
$$

for all $x, z \in E$. This implies that $\left\{8^{j} f\left(\frac{x}{2^{j}}, \frac{z}{2^{j}}\right)\right\}$ is Cauchy seqeunce in $X$ for all $x, z \in E$. By completeness of $X$, the sequence $\left\{8^{j} f\left(\frac{x}{2^{j}}, \frac{z}{2^{j}}\right)\right\}$ is convergent sequence for all $x, y \in E$. Define $F: E \times E \rightarrow X$ by

$$
F(x, z)=\lim _{j \rightarrow \infty} 8^{j} f\left(\frac{x}{2^{j}}, \frac{z}{2^{j}}\right)
$$

for all $x, z \in E$. By (2.21), we obtain that

$$
\begin{aligned}
& P(F(x+y, z+w)+F(x+y, z-w)-2 F(x, z)-2 F(x, w)-2 F(y, z)-2 F(y, w)) \\
= & P\left(\lim _{j \rightarrow \infty} 8^{j} f\left(\frac{x+y}{2^{j}}, \frac{z+w}{2^{j}}\right)+\lim _{j \rightarrow \infty} 8^{j} f\left(\frac{x+y}{2^{j}}, \frac{z-w}{2^{j}}\right)-2 \cdot \lim _{j \rightarrow \infty} 8^{j} f\left(\frac{x}{2^{j}}, \frac{z}{2^{j}}\right)\right. \\
& \left.-2 \cdot \lim _{j \rightarrow \infty} 8^{j} f\left(\frac{x}{2^{j}}, \frac{w}{2^{j}}\right)-2 \cdot \lim _{j \rightarrow \infty} 8^{j} f\left(\frac{y}{2^{j}}, \frac{z}{2^{j}}\right)-2 \cdot \lim _{j \rightarrow \infty} 8^{j} f\left(\frac{y}{2^{j}}, \frac{w}{2^{j}}\right)\right) \\
= & \lim _{j \rightarrow \infty} P\left(8^{j} f\left(\frac{x+y}{2^{j}}, \frac{z+w}{2^{j}}\right)+8^{j} f\left(\frac{x+y}{2^{j}}, \frac{z-w}{2^{j}}\right)-2 \cdot 8^{j} f\left(\frac{x}{2^{j}}, \frac{z}{2^{j}}\right)\right. \\
& \left.-2 \cdot 8^{j} f\left(\frac{x}{2^{j}}, \frac{w}{2^{j}}\right)-2 \cdot 8^{j} f\left(\frac{y}{2^{j}}, \frac{z}{2^{j}}\right)-2 \cdot 8^{j} f\left(\frac{y}{2^{j}}, \frac{w}{2^{j}}\right)\right) \\
\leq & \lim _{j \rightarrow \infty} 8^{j} P\left(f\left(\frac{x+y}{2^{j}}, \frac{z+w}{2^{j}}\right)+f\left(\frac{x+y}{2^{j}}, \frac{z-w}{2^{j}}\right)-2 f\left(\frac{x}{2^{j}}, \frac{z}{2^{j}}\right)-2 f\left(\frac{x}{2^{j}}, \frac{w}{2^{j}}\right)\right. \\
& \left.-2 f\left(\frac{y}{2^{j}}, \frac{z}{2^{j}}\right)-2 f\left(\frac{y}{2^{j}}, \frac{w}{2^{j}}\right)\right) \\
\leq & \lim _{j \rightarrow \infty} 8^{j} \varphi\left(\frac{x}{2^{j}}, \frac{y}{2^{j}}, \frac{z}{2^{j}}, \frac{w}{2^{j}}\right)=0
\end{aligned}
$$


for all $x, y, z, w \in E$. Since $X$ is total, we have

$$
F(x+y, z+w)+F(x+y, z-w)=2 F(x, z)+2 F(x, w)+2 F(y, z)+2 F(y, w)
$$

for all $x, y, z, w \in E$. Setting $l=0$ and letting $m \rightarrow \infty$ in (2.26), the inequality (2.26) holds. Next, we will show that $F$ is unique.

Let $G: E \times E \rightarrow X$ be a another mapping satisfying (1.2) and (2.22). It follows from Theorem 3 in [13] that there exists multi-additive mapping $M, M^{\prime}: E \times E \times E \rightarrow X$ such that $F(x, y)=M(x, y, y)$, $G(x, y)=M^{\prime}(x, y, y), M(x, y, z)=M(x, z, y)$ and $M^{\prime}(x, y, z)=M^{\prime}(x, z, y)$ for all $x, y, z \in E$. For any $n \in \mathbb{N}$, we get that

$$
\begin{aligned}
P(F(x, y)-G(x, y)) & =P\left(M(x, y, y)-M^{\prime}(x, y, y)\right) \\
& =P\left(M\left(\frac{2^{n} x}{2^{n}}, \frac{2^{n} y}{2^{n}}, \frac{2^{n} y}{2^{n}}\right)-M^{\prime}\left(\frac{2^{n} x}{2^{n}}, \frac{2^{n} y}{2^{n}}, \frac{2^{n} y}{2^{n}}\right)\right) \\
& =P\left(8^{n}\left[M\left(\frac{x}{2^{n}}, \frac{y}{2^{n}}, \frac{y}{2^{n}}\right)\right]-8^{n}\left[M^{\prime}\left(\frac{x}{2^{n}}, \frac{y}{2^{n}}, \frac{y}{2^{n}}\right)\right]\right) \\
& =P\left(8^{n}\left[M\left(\frac{x}{2^{n}}, \frac{y}{2^{n}}, \frac{y}{2^{n}}\right)-M^{\prime}\left(\frac{x}{2^{n}}, \frac{y}{2^{n}}, \frac{y}{2^{n}}\right)\right]\right) \\
& \leq 8^{n} P\left(M\left(\frac{x}{2^{n}}, \frac{y}{2^{n}}, \frac{y}{2^{n}}\right)-M^{\prime}\left(\frac{x}{2^{n}}, \frac{y}{2^{n}}, \frac{y}{2^{n}}\right)\right) \\
& \leq 8^{n} P\left(F\left(\frac{x}{2^{n}}, \frac{y}{2^{n}}\right)-f\left(\frac{x}{2^{n}}, \frac{y}{2^{n}}\right)+f\left(\frac{x}{2^{n}}, \frac{y}{2^{n}}\right)-G\left(\frac{x}{2^{n}}, \frac{y}{2^{n}}\right)\right) \\
& \leq 8^{n}\left[P\left(F\left(\frac{x}{2^{n}}, \frac{y}{2^{n}}\right)-f\left(\frac{x}{2^{n}}, \frac{y}{2^{n}}\right)\right)+P\left(f\left(\frac{x}{2^{n}}, \frac{y}{2^{n}}\right)\right.\right. \\
& \left.\left.-G\left(\frac{x}{2^{n}}, \frac{y}{2^{n}}\right)\right)\right] \\
& \leq 2 \cdot 8^{n} \tilde{\varphi}\left(\frac{x}{2^{n}}, \frac{x}{2^{n}}, \frac{y}{2^{n}}, \frac{y}{2^{n}}\right) \\
= & 2 \cdot 8^{n} \sum_{j=0}^{\infty} 8^{j} \varphi\left(\frac{\frac{x}{2^{n}}}{2^{j+1}}, \frac{\frac{x}{2^{n}}}{2^{j+1}}, \frac{\frac{y}{2^{n}}}{2^{j+1}}, \frac{\frac{y}{2^{n}}}{2^{j+1}}\right) \\
= & 2 \cdot \sum_{j=0}^{\infty} 8^{n+j} \varphi\left(\frac{x}{2^{n+j+1}}, \frac{x}{2^{n+j+1}}, \frac{y}{2^{n+j+1}}, \frac{y}{2^{n+j+1}}\right) \\
= & 2 \cdot \sum_{i=n}^{\infty} 8^{i} \varphi\left(\frac{x}{2^{i+1}}, \frac{x}{2^{i+1}}, \frac{y}{2^{i+1}}, \frac{y}{2^{i+1}}\right)
\end{aligned}
$$

for all $x, y \in E$. By (2.23), we get that

$$
\lim _{n \rightarrow \infty} \sum_{i=n}^{\infty} 8^{i} \varphi\left(\frac{x}{2^{i+1}}, \frac{x}{2^{i+1}}, \frac{y}{2^{i+1}}, \frac{y}{2^{i+1}}\right)=0
$$

for all $x, y \in E$. Hence

$$
\lim _{n \rightarrow \infty} P(F(x, y)-G(x, y))=0
$$

for all $x, y \in E$. Since paranorm $P$ on $X$ is total, we have $F(x, y)-G(x, y)=0$ for all $x, y \in E$. Hence $F$ is a unique mapping satisfying (1.2) and (2.22). 
Remark 2.2. Let $r, \theta$ be positive real numbers with $r>3$. If we set $\varphi(x, y, z, w)=\theta\left(\|x\|^{r}+\|y\|^{r}+\|z\|^{r}+\right.$ $\left.\|w\|^{r}\right)$ for all $x, y, z, w \in E$, then Theorem 2.2 recovers Theorem 3.1 in [2].

3. Stability of the generalized Cauchy-Jensen functional equation in paranormed space

The following result is the Hyers-Ulam-Rassias stability of the functional equation (1.5).

Theorem 3.1. Let $r$ be a positive real number with $r>1$, and let $f: E \rightarrow X$ be a mapping satisfying

$$
P\left(\alpha f\left(\frac{x+y}{\alpha}+z\right)-f(x)-f(y)-\alpha f(z)\right) \leq \theta\left(\|x\|^{r}+\|y\|^{r}+\|z\|^{r}\right)
$$

for all $x, y, z \in E$. Then there exists a unique mapping $F: E \rightarrow X$ satisfying (1.5) such that

$$
P(f(x)-F(x)) \leq\left(\frac{3 \alpha^{r}+1}{\alpha^{r}-\alpha}\right) \theta\|x\|^{r}
$$

for all $x \in E$ where the mapping $F: E \rightarrow X$ is given by

$$
F(x)=\lim _{n \rightarrow \infty} \alpha^{n} f\left(\frac{x}{\alpha^{n}}\right)
$$

for all $x \in E$.

Proof. Putting $x=y=z=0$ in (3.1), we have $P(f(0)) \leq 0$. Since $X$ is total, we obtain that $f(0)=0$. Letting $x=-\frac{x}{\alpha}, y=\frac{x}{\alpha}$ and $z=0$ in (3.1), we obtain that

$$
\begin{aligned}
& P\left(f\left(-\frac{x}{\alpha}\right)+f\left(\frac{x}{\alpha}\right)\right) \\
= & P\left(-f\left(-\frac{x}{\alpha}\right)-f\left(\frac{x}{\alpha}\right)\right) \\
= & P\left(\alpha f\left(\frac{-\frac{x}{\alpha}+\frac{x}{\alpha}}{\alpha}+0\right)-f\left(-\frac{x}{\alpha}\right)-f\left(\frac{x}{\alpha}\right)-\alpha f(0)\right) \\
\leq & \theta\left(\left\|-\frac{x}{\alpha}\right\|^{r}+\left\|\frac{x}{\alpha}\right\|^{r}+\|0\|^{r}\right) \\
= & \frac{2 \theta}{\alpha^{r}}\|x\|^{r}
\end{aligned}
$$

for all $x \in E$. Replacing $x$ by $\alpha x$ in the inequality (3.3), we get that

$$
P(f(-x)+f(x)) \leq 2 \theta\|x\|^{r}
$$


for all $x \in E$. Replacing $x=-x, y=0$, and $z=\frac{x}{\alpha}$ in (3.1), we have

$$
\begin{aligned}
& P\left(f(-x)+\alpha f\left(\frac{x}{\alpha}\right)\right) \\
= & P\left(-f(-x)-\alpha f\left(\frac{x}{\alpha}\right)\right) \\
= & P\left(\alpha f\left(\frac{-x+0}{\alpha}+\left(-\frac{x}{\alpha}\right)\right)-f(-x)-f(0)-\alpha f\left(\frac{x}{\alpha}\right)\right) \\
\leq & \theta\left(\|-x\|^{r}+\|0\|^{r}+\left\|\frac{x}{\alpha}\right\|^{r}\right) \\
= & \left(1+\frac{1}{\alpha^{r}}\right) \theta\|x\|^{r}
\end{aligned}
$$

for all $x \in E$. It follows from (3.4) and (3.5) that we have

$$
\begin{aligned}
P\left(\alpha f\left(\frac{x}{\alpha}\right)-f(x)\right) & =P\left(\alpha f\left(\frac{x}{\alpha}\right)+f(-x)-f(-x)-f(x)\right) \\
& \leq P\left(\alpha f\left(\frac{x}{\alpha}\right)+f(-x)\right)+P(f(-x)+f(x)) \\
& \leq\left(1+\frac{1}{\alpha^{r}}\right) \theta\|x\|^{r}+2 \theta\|x\|^{r} \\
& \leq\left(3+\frac{1}{\alpha^{r}}\right) \theta\|x\|^{r}
\end{aligned}
$$

for all $x \in E$. For $i \in \mathbb{N}$, replacing $x=\frac{x}{\alpha^{i}}$ in (3.6), we get that

$$
\begin{aligned}
P\left(\alpha^{i+1} f\left(\frac{x}{\alpha^{i+1}}\right)-\alpha^{i} f\left(\frac{x}{\alpha^{i}}\right)\right) & \leq \alpha^{i} P\left(\alpha f\left(\frac{x}{\alpha^{i+1}}\right)-f\left(\frac{x}{\alpha^{i}}\right)\right) \\
& \leq \alpha^{i}\left(3+\frac{1}{\alpha^{r}}\right) \theta\left\|\frac{x}{\alpha^{i}}\right\|^{r} \\
& =\left(\frac{1}{\alpha^{r-1}}\right)^{i}\left(3+\frac{1}{\alpha^{r}}\right) \theta\|x\|^{r}
\end{aligned}
$$

for all $x \in E$. For given nonnegative integer $l, m$ such that $l<m$, we have

$$
\begin{aligned}
& P\left(\alpha^{m} f\left(\frac{x}{\alpha^{m}}\right)-\alpha^{l} f\left(\frac{x}{\alpha^{l}}\right)\right) \\
= & P\left(\alpha^{m} f\left(\frac{x}{\alpha^{m}}\right)-\alpha^{m-1} f\left(\frac{x}{\alpha^{m-1}}\right)+\alpha^{m-1} f\left(\frac{x}{\alpha^{m-1}}\right)\right. \\
& \left.+\cdots+\alpha^{l+1} f\left(\frac{x}{\alpha^{l+1}}\right)-\alpha^{l} f\left(\frac{x}{\alpha^{l}}\right)\right) \\
\leq & \sum_{j=l}^{m-1} P\left(\alpha^{j+1} f\left(\frac{x}{\alpha^{j+1}}\right)-\alpha^{j} f\left(\frac{x}{\alpha^{j}}\right)\right) \\
\leq & \sum_{j=l}^{m-1}\left(\frac{1}{\alpha^{r-1}}\right)^{i}\left(3+\frac{1}{\alpha^{r}}\right) \theta\|x\|^{r} \\
\leq & \left(3+\frac{1}{\alpha^{r}}\right) \theta\|x\|^{r} \sum_{j=0}^{\infty}\left(\frac{1}{\alpha^{r-1}}\right)^{i}
\end{aligned}
$$


for all $x \in E$. Since $r>1$, we have $\frac{1}{\alpha^{r-1}}<1$. Since $\frac{1}{\alpha^{r-1}}<1$, the sequence $\left\{\alpha^{n} f\left(\frac{x}{\alpha^{n}}\right)\right\}$ is Cauchy sequence for all $x \in E$. By completeness of $X$, the sequence $\left\{\alpha^{n} f\left(\frac{x}{\alpha^{n}}\right)\right\}$ converges. Define $F: E \rightarrow X$ by

$$
F(x)=\lim _{n \rightarrow \infty} \alpha^{n} f\left(\frac{x}{\alpha^{n}}\right)
$$

for all $x \in E$. Moreover, letting $l=0$ and taking limit $m \rightarrow \infty$ in (3.8), we can obtain that inequality (3.2). It follows from (3.1) and (3.9) that

$$
\begin{aligned}
& P\left(\alpha F\left(\frac{x+y}{\alpha}+z\right)-F(x)-F(y)-\alpha F(z)\right) \\
= & P\left(\alpha \cdot \lim _{n \rightarrow \infty} \alpha^{n} f\left(\frac{\frac{x+y}{\alpha}+z}{\alpha^{n}}\right)-\lim _{n \rightarrow \infty} \alpha^{n} f\left(\frac{x}{\alpha^{n}}\right)-\lim _{n \rightarrow \infty} \alpha^{n} f\left(\frac{y}{\alpha^{n}}\right)\right. \\
& \left.-\alpha \lim _{n \rightarrow \infty} \alpha^{n} f\left(\frac{z}{\alpha^{n}}\right)\right) \\
= & \lim _{n \rightarrow \infty} \alpha^{n} P\left(\alpha f\left(\frac{\frac{x}{\alpha^{n}}+\frac{y}{\alpha^{n}}}{\alpha}+\frac{z}{\alpha^{n}}\right)-f\left(\frac{x}{\alpha^{n}}\right)-f\left(\frac{y}{\alpha^{n}}\right)-\alpha f\left(\frac{z}{\alpha^{n}}\right)\right) \\
\leq & \lim _{n \rightarrow \infty} \alpha^{n} \theta\left(\left\|\frac{x}{\alpha^{n}}\right\|^{r}+\left\|\frac{x}{\alpha^{n}}\right\|^{r}+\left\|\frac{x}{\alpha^{n}}\right\|^{r}\right) \\
= & \theta\|x\|^{r} \lim _{n \rightarrow \infty}\left(\frac{1}{\alpha^{r-1}}\right)^{n}=0
\end{aligned}
$$

for all $x, y, z \in E$. Since $X$ is total, we have

$$
\alpha F\left(\frac{x+y}{\alpha}+z\right)=F(x)+F(y)+\alpha F(z)
$$

for all $x, y, z \in E$. By Corollary 1.1, $F$ is additive. Next, we will show that $F$ is unique. Let $G$ be another mapping satisfying (1.5) and (3.2). Then, we consider

$$
\begin{aligned}
P(F(x)-G(x)) & =P\left(n F\left(\frac{x}{n}\right)-n f\left(\frac{x}{n}\right)+n f\left(\frac{x}{n}\right)-n G\left(\frac{x}{n}\right)\right) \\
& \leq n\left(P\left(F\left(\frac{x}{n}\right)-f\left(\frac{x}{n}\right)\right)+P\left(f\left(\frac{x}{n}\right)-G\left(\frac{x}{n}\right)\right)\right) \\
& \leq 2 n \theta\left(\frac{3 \alpha^{r}+1}{\alpha^{r}-\alpha}\right)\left\|\frac{x}{n}\right\|^{r} \\
& =\left(\frac{1}{n^{r-1}}\right) 2 \theta\left(\frac{3 \alpha^{r}+1}{\alpha^{r}-\alpha}\right)\|x\|^{r}
\end{aligned}
$$

for all $x \in E$. Since $r-1>0$, taking limit $n \rightarrow \infty$ in $(3.10)$, we have $P(F(x)-G(x))=0$ for all $x \in E$. Since $X$ is total, we have $F(x)=G(x)$ for all $x \in E$, that is $F$ is unique.

Theorem 3.2. Let $r$ be a positive real number with $r<1$ and let $f: X \rightarrow E$ be a mapping satisfying

$$
\left\|\alpha f\left(\frac{x+y}{\alpha}+z\right)-f(x)-f(y)-\alpha f(z)\right\| \leq P(x)^{r}+P(y)^{r}+P(z)^{r}
$$

for all $x, y, z \in X$. Then there exists a unique mapping $F: X \rightarrow E$ satisfying (1.5) such that

$$
\|f(x)-F(x)\| \leq \frac{2+3 \alpha^{r}}{\alpha-\alpha^{r}} P(x)^{r}
$$


for all $x \in X$ where the mapping $F: X \rightarrow E$ is given by

$$
F(x)=\lim _{n \rightarrow \infty} \frac{1}{\alpha^{n}} f\left(\alpha^{n} x\right)
$$

for all $x \in X$.

Proof. Letting $x=y=z=0$ in (3.11), we get that

$$
\begin{aligned}
\|2 f(0)\| & =\left\|\alpha f\left(\frac{0+0}{\alpha}+0\right)-f(0)-f(0)-\alpha f(0)\right\| \\
& \leq P(0)^{r}+P(0)^{r}+P(0)^{r} \\
& =0
\end{aligned}
$$

So $f(0)=0$. Subtituting $x=-\alpha x, y=0$ and $z=x$ in (3.11), we obtain that

$$
\begin{aligned}
\|f(-\alpha x)+\alpha f(x)\| & =\left\|\alpha f\left(\frac{-\alpha x+0}{\alpha}+x\right)-f(-\alpha x)-f(0)-\alpha f(x)\right\| \\
& \leq P(-\alpha x)^{r}+P(0)^{r}+P(x)^{r} \\
& \leq\left(1+\alpha^{r}\right) P(x)^{r}
\end{aligned}
$$

for all $x \in X$. Letting $x=-\alpha x, y=\alpha x$ and $z=x$, we get that

$$
\begin{aligned}
\|f(-\alpha x)+f(\alpha x)\| & =\left\|\alpha f\left(\frac{-\alpha x+\alpha x}{\alpha}+x\right)-f(-\alpha x)-f(\alpha x)-\alpha f(x)\right\| \\
& \leq P(-\alpha x)^{r}+P(\alpha x)^{r}+P(x)^{r} \\
& \leq\left(1+2 \alpha^{r}\right) P(x)^{r}
\end{aligned}
$$

for all $x \in X$. Then we have

$$
\begin{aligned}
\|f(\alpha x)-\alpha f(x)\| & =\|f(\alpha x)+f(-\alpha x)-f(-\alpha x)-\alpha f(x)\| \\
& =\|f(\alpha x)+f(-\alpha x)\|+\|f(-\alpha x)+\alpha f(x)\| \\
& \leq\left(1+\alpha^{r}\right) P(x)^{r}+\left(1+2 \alpha^{r}\right) P(x)^{r} \\
& =\left(2+3 \alpha^{r}\right) P(x)^{r}
\end{aligned}
$$

and so

$$
\left\|\frac{1}{\alpha} f(\alpha x)-f(x)\right\| \leq \frac{2+3 \alpha^{r}}{\alpha} P(x)^{r}
$$

for all $x \in X$. Replacing $x=\alpha^{i} x$ and multiplying by $\frac{1}{\alpha^{i}}$ in (3.13), we have

$$
\begin{aligned}
\left\|\frac{1}{\alpha^{i+1}} f\left(\alpha^{i+1} x\right)-\frac{1}{\alpha^{i}} f\left(\alpha^{i} x\right)\right\| & \leq \frac{1}{\alpha^{i}} \cdot \frac{2+3 \alpha^{r}}{\alpha} P\left(\alpha^{i} x\right)^{r} \\
& \leq \frac{2+3 \alpha^{r}}{\alpha} P(x)^{r} \cdot\left(\frac{1}{\alpha^{1-r}}\right)^{i}
\end{aligned}
$$


for all $x \in X$. By (3.14), for any integers $l, m$ such that $0 \leq l<m$, we obtain that

$$
\begin{aligned}
& \left\|\frac{1}{\alpha^{m}} f\left(\alpha^{m} x\right)-\frac{1}{\alpha^{l}} f\left(\alpha^{l} x\right)\right\| \\
= & \| \frac{1}{\alpha^{m}} f\left(\alpha^{m} x\right)+\frac{1}{\alpha^{m-1}} f\left(\alpha^{m-1} x\right)-\frac{1}{\alpha^{m-1}} f\left(\alpha^{m-1} x\right)+\cdots+\frac{1}{\alpha^{l+1}} f\left(\alpha^{l+1} x\right) \\
& -\frac{1}{\alpha^{l}} f\left(\alpha^{l} x\right) \| \\
\leq & \sum_{i=l}^{m-1} \frac{2+3 \alpha^{r}}{\alpha} P(x)^{r} \cdot\left(\frac{1}{\alpha^{1-r}}\right)^{i} \\
\leq & \frac{2+3 \alpha^{r}}{\alpha} P(x)^{r} \cdot \sum_{i=0}^{\infty}\left(\frac{1}{\alpha^{1-r}}\right)^{i}
\end{aligned}
$$

for all $x \in X$. Since $\frac{1}{\alpha^{1-r}}<1$, we have $\sum_{i=0}^{\infty}\left(\frac{1}{\alpha^{1-r}}\right)^{i}<\infty$. It follows from (3.15) that the sequence $\left\{\frac{1}{\alpha^{n}} f\left(\alpha^{n} x\right)\right\}$ is Cauchy sequence for all $x \in X$. Since $E$ is complete, the sequence $\left\{\frac{1}{\alpha^{n}} f\left(\alpha^{n} x\right)\right\}$ is convergent sequence. We define a mapping $F: X \rightarrow E$ by

$$
F(x)=\lim _{n \rightarrow \infty} \frac{1}{\alpha^{n}} f\left(\alpha^{n} x\right)
$$

for all $x \in X$. Moreover, letting $l=0$ and taking limit $m \rightarrow \infty$ in (3.15), we can obtain that inequality (3.12). It follows from (3.11) and (3.16) that we have

$$
\begin{aligned}
& \left\|\alpha F\left(\frac{x+y}{\alpha}+z\right)-F(x)-F(y)-\alpha F(z)\right\| \\
= & \| \alpha \lim _{n \rightarrow \infty} \frac{1}{\alpha^{n}} f\left(\alpha^{n}\left(\frac{x+y}{\alpha}+z\right)\right)-\lim _{n \rightarrow \infty} \frac{1}{\alpha^{n}} f\left(\alpha^{n} y\right)-\lim _{n \rightarrow \infty} \frac{1}{\alpha^{n}} f\left(\alpha^{n} z\right) \\
& -\alpha \lim _{n \rightarrow \infty} \frac{1}{\alpha^{n}} f\left(\alpha^{n} z\right) \| \\
\leq & \lim _{n \rightarrow \infty} \frac{1}{\alpha^{n}}\left\|\alpha f\left(\frac{\alpha^{n} x+\alpha^{n} y}{\alpha}+\alpha^{n} z\right)-f\left(\alpha^{n} y\right)-f\left(\alpha^{n} z\right)-\alpha f\left(\alpha^{n} z\right)\right\| \\
\leq & \lim _{n \rightarrow \infty} \frac{1}{\alpha^{n}}\left\|\alpha f\left(\frac{\alpha^{n} x+\alpha^{n} y}{\alpha}+\alpha^{n} z\right)-f\left(\alpha^{n} y\right)-f\left(\alpha^{n} z\right)-\alpha f\left(\alpha^{n} z\right)\right\| \\
\leq & \lim _{n \rightarrow \infty} \frac{1}{\alpha^{n}}\left(P\left(\alpha^{n} x\right)^{r}+P\left(\alpha^{n} y\right)^{r}+P\left(\alpha^{n} z\right)^{r}\right) \\
\leq & \lim _{n \rightarrow \infty} \frac{\alpha^{n r}}{\alpha^{n}}\left(P(x)^{r}+P(y)^{r}+P(z)^{r}\right) \\
\leq & \left(P(x)^{r}+P(y)^{r}+P(z)^{r}\right) \lim _{n \rightarrow \infty}\left(\frac{1}{\alpha^{1-r}}\right)^{n}=0
\end{aligned}
$$

for all $x, y, z \in X$. Hence

$$
\alpha F\left(\frac{x+y}{\alpha}+z\right)=F(x)+F(y)+\alpha F(z)
$$

for all $x, y, z \in X$, that is $F$ is the generalized Cauchy-Jensen functional equation. By tha same reasoning as in the proof of Theorem 3.1, $F$ is unique.

Competing interests. The author declare that they have no competing interests. 
Author's contributions. All authors contributed equally to writing of this paper. All authors read and approved the final manuscript.

Acknowledgement. The authors would like to thank Naresuan university and Science Achievement Scholarship of Thailand, which provides facilities and funding for this research.

\section{REFERENCES}

[1] J.H. Bae and W.G. Park, Approximate quadratic forms in paranormed spaces, J. Comput. Anal. Appl., 19 (4) (2015), $740-750$

[2] J.H. Bae and W.G. Park, On the Ulam stability of the Cauchy-Jensen equation and the additive-quadratic equation, J. Nonlinear Sci. Appl., 8 (2015), 710-718

[3] J. Brzdęk, W. Fechner, M.S. Moslehian and J. Silorska, Recent developments of the conditional stability of the homomorphism equation, Banach J. Math. Anal., 9 (2015), 278-326.

[4] J. Brzdęk, D. Popa, I. Rasa and B. Xu, Ulam Stability of Operators, Mathematical Analysis and its Applications vol.1, Academic Press, Elsevier, Oxford, 2018.

[5] Z.X. Gao, H.X. Cao, W.T. Zheng and L. Xu, Generalized Hyers-Ulam-Rassias stability of functional inequalities and functional equations, J. Math. Inequal., 3 (1) (2009) 63-77

[6] P. Găvruța, A generalization of the Hyers-Ulam-Rassias stability of approximately additive mapping, J. Math. Anal. Appl., 184 (1994), 431-436

[7] D.H. Hyers, On the stability of the linear functional equation, Proceedings of the National Academy of Sciences of the United States of America, 27 (4) (1941), 222-224

[8] K.W. Jun, Y.H. Lee and Y.S. Cho, On the stability of Cauchy-Jensen functional equation, Commun. Korean Math. Soc., 23 (3) (2008) 377-386

[9] K.W. Jun, Y.H. Lee and J.A. Son, On the stability of Cauchy-Jensen functional equation III, Korean J. Math., 16 (2) (2008) 205-214

[10] M. Kuczma, An Introduction to the Theory of Functional Equational Equations and Inequalties: Cauchy's Equation and Jensen's Inequality, Birkhäuser Verlag Basel, 2009.

[11] S.J. Lee, C. Park and J.R. Lee, Functional inequalities in paranormed spaces, J. Chungcheong Math. Soc., 262 (2013), $287-296$.

[12] W.G. Park and J.H. Bae, On a Cauchy-Jensen functional equation and its stability, J. Math.Anal. Appl., 323 (1) (2006), $634-643$

[13] W.G. Park, J.H. Bae and B.H. Chung, On an additive-quadratic functional equation and its stability, J. Appl. Math. \& Computing, 18 (1-2) (2005), 563-572

[14] C. Park and J.R. Lee, Functional equations and inequalities in paranormed spaces, J. Inequal. Appl., 2013 (2013), Art. ID 198.

[15] C. Park and D.Y. Shin, Functional equations in paranormed spaces, Adv. Differ. Equ., 2012 (2012), Art. ID 123.

[16] Th.M. Rassias, On the stability of the linear mapping in Banach spaces, Proc. Amer. Math. Soc. 72 (2) (1978), 297-300

[17] S.M. Ulam, A Collection of Mathematical Problems, Interscience Tracts in Pure and AppliedMathematics, no. 8, Interscience Publishers, New York, NY, USA, 1960.

[18] A. Wilansky, Modern Methods in Topological Vector Space, McGraw-Hill International Book Co., New York, 1, 1978. 